\title{
Gastrointestinal mucosal barrier function and diseases
}

\author{
Tadayuki Oshima ${ }^{1} \cdot$ Hiroto Miwa ${ }^{1}$
}

Received: 25 March 2016/Accepted: 29 March 2016/Published online: 5 April 2016

(c) Japanese Society of Gastroenterology 2016

\begin{abstract}
The gastrointestinal mucosal barrier plays an essential role in the separation of the inside of the body from the outside environment. Tight junctions (TJs) are the most important component for construction of a constitutive barrier of epithelial cells, and they regulate the permeability of the barrier by tightly sealing the cell-cell junctions. TJ proteins are represented by claudins, occludin, junctional adhesion molecules, and scaffold protein zonula occludens. Among these TJ proteins, claudins are the major components of TJs and are responsible for the barrier and the polarity of the epithelial cells. Gastrointestinal diseases including reflux esophagitis, inflammatory bowel disease, functional gastrointestinal disorders, and cancers may be regulated by these molecules, and disruption of their functions leads to chronic inflammatory conditions and chronic or progressive disease. Therefore, regulation of the barrier function of epithelial cells by regulating the expression and localization of TJ proteins is a potential new target for the treatment of these diseases. Treatment strategies for these diseases might thus be largely altered if symptom generation and/or immune dysfunction could be regulated through improvement of mucosal barrier function. Since TJ proteins may also modify tumor infiltration and metastasis, other important goals include finding a good TJ biomarker of cancer progression and patient prognosis, and developing TJ protein-targeted therapies that can modify patient prognosis. This review summarizes current understanding of gastrointestinal barrier function, TJ protein expression, and
\end{abstract}

Tadayuki Oshima

t-oshima@hyo-med.ac.jp

1 Division of Gastroenterology, Department of Internal Medicine, Hyogo College of Medicine, 1-1 Mukogawa-cho, Nishinomiya, Hyogo 663-8501, Japan the mechanisms underlying epithelial barrier dysregulation in gastrointestinal diseases.

Keywords Claudin Permeability - Tight junction . Esophagitis · Cancer · Irritable bowel syndrome

\section{Introduction}

The gastrointestinal mucosal barrier plays an essential role in the separation of the inside of the body from the outside environment. Tight junctions (TJs) are the most important structural component for the formation of constitutive barrier function in epithelial cells. The functions of TJs are described by their barrier and fence functions. TJs present on the apical end of the lateral membrane surface in columnar epithelial cells form a barrier against paracellular transport and maintain apicobasal cell polarity through their fence function [1, 2].

In the late 1990s, new TJ molecules were identified as surface-expressed core proteins, including claudins (CLDNs), occludin, and junctional adhesion molecules (JAMs), and these molecules bind directly to the scaffold protein zonula occludens (ZOs) $[3,4]$. These molecules are expressed on both epithelial and endothelial cells in different expression patterns.

Occludin was first described as a TJ molecule incorporated directly into TJ strands. However, it does not contribute to direct TJ barrier function [5-7]. CLDNs are small tetraspanning transmembrane proteins with sizes ranging from 20 to $27 \mathrm{kDa}$, and at least 27 types of CLDNs have been identified in humans [8]. These claudin subtypes are expressed in an organ-specific manner and regulate the tissue-specific physiological functions of TJs [4]. Based on sequence similarity, CLDN1-10, 14, 15, 17 and 19 are 
called classic CLDNs, and CLDN-11, 12, 13, 16, 18, 29 and 24 are called non-classic CLDNs [9].

Following the identification of these surface-expressed $\mathrm{TJ}$ proteins, their involvement in the function of TJs, including in the GI tract, was gradually revealed, and CLDNs are now known to be the major protein components of TJs regulating paracellular permeability. Furthermore, the loss of TJs can account for cancer progression that might be due to the loss of cell-cell adhesion and cell differentiation. Recent reports have indicated that both the downregulation and upregulation of $\mathrm{TJ}$ proteins influence cancer progression and patient prognosis [10]. However, the results are still difficult to interpret, and the published data are confusing.

This review explores the expression pattern and the localization of TJ proteins in the GI tract (Fig. 1). The upregulation, downregulation, and delocalization of TJ proteins in gastrointestinal diseases and the clinical significance of these molecules are also discussed.

\section{TJs in the esophagus}

The history of research into gastrointestinal intestinal barrier function and $\mathrm{TJ}$ molecules, especially in the esophagus, is short. Until recently, it was largely acknowledged, based on a statement in a pathology textbook published in 1994, that "stratified squamous epithelium is somewhat atypical in that junctional complexes are not found" [11]. However, recent molecular techniques and identification of new TJ proteins have revealed the existence of TJs in esophageal epithelial layers. Both the esophagus and skin consist of stratified epithelial cell layers, and while they differ

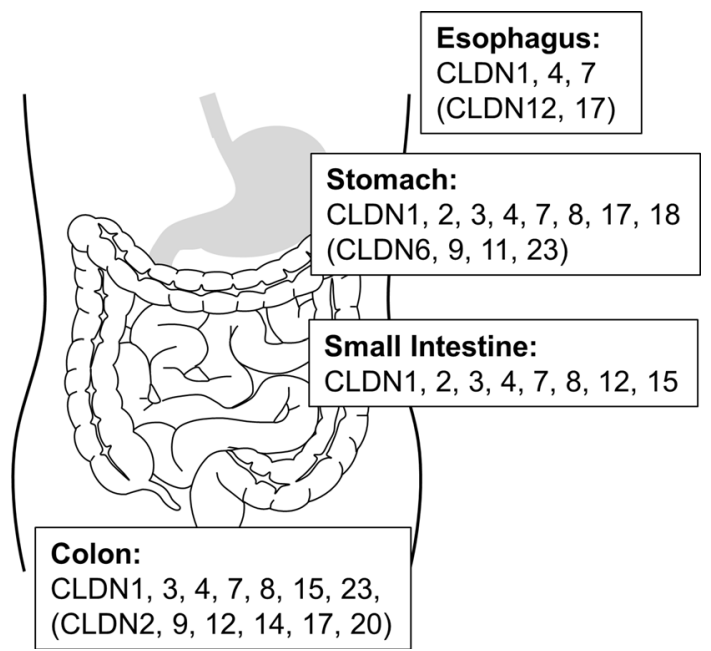

Fig. 1 Expression patterns of claudin (CLDN) proteins in the gastrointestinal tract. Claudins expressed in the esophagus, stomach, small intestine, and colon are indicated. Expression of the CLDNs in parentheses can be modulated in diseases significantly in terms of the existence of keratinization, aside from keratinization, the esophageal mucosal epithelial layers must be similar to skin in having TJs. Based on skin data [6], therefore, TJ strands should exist in granular layers. However, recent exploration of TJ proteins has also revealed the expression of $\mathrm{TJ}$ proteins in esophageal mucosal layers. Expression of occludin specifically indicates the location of TJs, and occludin staining of human esophageal tissue was limited to granular layers, expressed as dots and whisker lines. Occludin was not expressed in basal layers (Fig. 2) [12]. These findings indicate that the localization of TJs in the esophagus is different from the location of dilated intercellular spaces (DISs), which will be discussed in the following section, and which are important for determining the real barrier of the esophagus.

\section{Lessons from in vitro three-dimensional esophageal cell culture models}

In evaluating the function of esophageal epithelial layers, we originally established esophageal stratified epithelial cell layers in vitro [12-16]. When we observed the development of stratified epithelial layers, we found that the barrier was dramatically developed 5 to 7 days after air-liquid interface (ALI)-culture of primary human squamous epithelial cells [15]. We also examined cell morphological changes at these time points. When granular layers started to develop, the TJ proteins occludin and ZO1 began to be expressed in these layers as dots and whisker lines $[14,15]$. These data are consistent with the localization of these TJ proteins in granular layers in the human esophagus. The granular layers that express occludin have a barrier function and regulate esophageal permeability. In other words, they are real TJ strands.

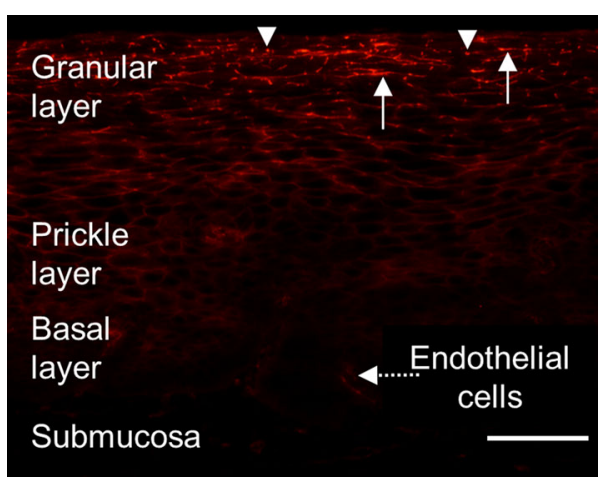

Fig. 2 Immunofluorescence staining of occludin in the human esophagus. Granular layers stain positive for occludin (red). Occludin is densely stained as dots (arrowheads) or whisker lines (arrows) in the granular layers but is not stained in the prickle cell or basal layers. Endothelial cells (arrow with dotted line) also stain positive for occludin. Bar $100 \mu \mathrm{m}$ 
We further demonstrated that stratified layers representing basal and prickle cell layers began to develop after 3 days of culture, and staining showed that these layers were lined with CLDN1 and CLDN4. However, a barrier function was totally absent in the layers on these days, indicating that expression of these TJ proteins and their localization as a lining of the cell membrane of the layers do not have a barrier function [14, 15]. The data also indicate that the DIS located at the basal and suprabasal layers does not contribute to the specific function of mucosal permeability (Fig. 3). CLDN1 and CLDN4 are stained as dense dots and whisker lines in granular layers, and show a barrier function when granular layers exist [13$15,17]$. It is reasonable to assume that layers showing such dense staining of TJ proteins in these cultures have functioning TJs, in a manner similar to that in monolayer cells. Staining of CLDN3 on monolayer cells shows dense staining at the apical lateral side, which has a barrier function, and lined staining at the lateral side of the membrane, which does not have barrier function (Fig. 4).

Although the TJ strands of monolayer cells can be detected by electron microscopy as tight attachments of cells at the apical lateral side of the cells, tight attachment of esophageal stratified cell layers is not easily detected even by electron microscopy. These data may explain why previous textbooks indicated the absence of TJs in the esophagus.

\section{The interpretation of $\mathbf{T J}$ formation and expression patterns}

Although there has been much interest in and a large amount of data generated regarding the recent discovery of TJ proteins and related proteins, interpretation of these data is difficult, and the data are confusing. Several factors

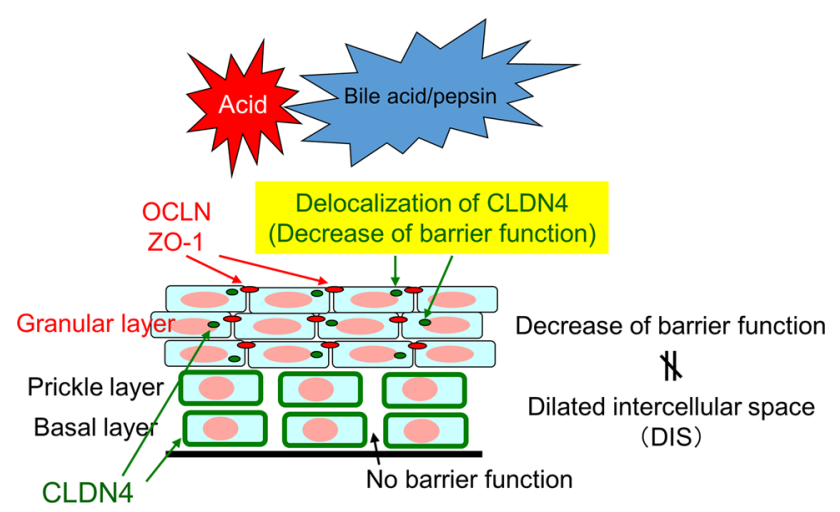

Fig. 3 Scheme of the esophageal tight junction (TJ) barrier including the localization of occludin, ZO-1, and CLDN4, and the dilated intercellular space (DIS)

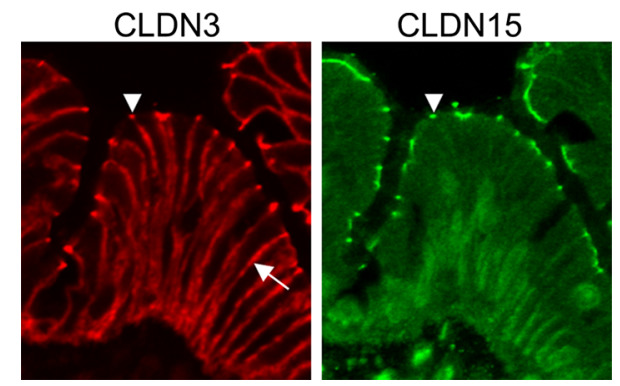

Fig. 4 Immunofluorescence staining of CLDN3 and CLDN15 in the human duodenum. In duodenal epithelia, CLDN3 is strongly stained at apical lateral membranes (arrowhead) and is also stained at lateral membranes (arrow). CLDN15 is strongly stained at apical lateral membranes (arrowhead) but is not stained at lateral membranes

explain why these controversial data continue to be published.

1. Intercellular junctions exist at the most apical side of the lateral membrane of columnar epithelial cells of the stomach and the colon, and TJs are recognized here by electron microscopy as close cell-cell contacts. Since occludin or ZO-1 can be specifically stained only at this location, such $\mathrm{TJ}$ s are easily recognized following immunofluorescence staining. However, claudins that function as the major TJ protein at the most apical surface of these cells not only can be densely stained at the apical lateral membrane, but are also widely stained at the lateral membrane of columnar epithelial cells. Therefore, even though TJs do not exist at the lateral membrane, CLDNs are stained on the lateral membrane. These data indicate that the surface staining of some CLDNs does not represent TJ strand formation (Fig. 4). These findings can be applied even to esophageal stratified epithelial cell layers.

2. Many papers discuss the expression of CLDN5 on epithelial cells, and these data were obtained by staining with so-called specific antibodies. However, these antibodies must have cross-reactivity with other antigens, since CLDN5 mRNA is rarely expressed by epithelial cells. Therefore, CLDN5 should be considered as a TJ protein of endothelial and not epithelial cells [18].

3. Complicated expression patterns of various CLDNs in different species make the data confusing. For example, CLDN3 was detected in a rat esophagus, and it has been reported to be modulated in rat esophageal disorders including reflux esophagitis [19-22]. However, CLDN3 is not detectable in the human esophagus, suggesting that, if it is expressed, it must be expressed at a very low level. These controversies may arise due to differences in the species used and to the 
specificity of the anti-claudin antibodies. However, the precise reasons for these differences remain to be elucidated, and further experiments are warranted.

4. The expression of TJ proteins is often determined based on immunohistochemical staining. However, the localization of $\mathrm{TJ}$ proteins is indicated differently by different antibodies, suggesting that these antibodies may have different reactivity and specificity. Thus, the level of mRNA and the staining of TJ antigens at times may not be correlated. Furthermore, when the protein amount of TJ antigens is examined in relation to the total protein amount, as mentioned in 1 above, TJ antigen expression itself is not always related to the strand formation of TJs. In this regard, when TJ-related proteins that function as TJs are evaluated, the amount of the detergent-insoluble fraction of $\mathrm{TJ}$ proteins should be evaluated, because TJ-forming proteins are detergent-insoluble [23]. However, few studies have performed such appropriate evaluation.

\section{Involvement of $\mathbf{T J}$ proteins in diseases}

\section{Gastroesophageal reflux disease (GERD)}

Esophageal mucosa consists of stratified epithelial layers and functions as a firm barrier against luminal stimulants including acids and bile acid. Disruption of this barrier leads to the development of reflux symptoms by reflux stimulants. The most prevalent type of GERD, non-erosive reflux disease (NERD), has shown DIS at the basal side of the stratified epithelial cell layers even without gross mucosal injury. DIS can be detected in the basal and prickle cell layers [24], and these phenomena are only detected in reflux esophagitis (RE) and NERD, and not in functional heartburn (FH) [25-27]. Farre et al. [28] also reported that when intercellular space was measured after weak acid, acid, and acidic bile perfusion, DIS was seen after the perfusion of weak acid, acid, and acidic bile but not of saline. The degree of DIS was not different among weak acid, acid and acidic bile. These data indicate that GER actually induces DIS. The data also showed that GER symptoms were not produced when DIS was seen in a healthy volunteer, suggesting that GER and DIS are not sufficient for the development of GERD symptoms.

In a rat model, stress induced DIS and increased ${ }^{51} \mathrm{Cr}$ EDTA permeability, indicating a relationship between DIS and esophageal permeability; the authors concluded that DIS induced esophageal mucosal permeability [29]. However, as mentioned above, DIS itself is not directly involved in increased permeability. Based on our experience with an ALI-cultured esophageal stratified epithelial model, DIS and increased permeability can be considered as two simultaneous but distinct and independent changes (Fig. 3).

We further examined the effects of acid and bile acids on epithelial barrier function using an in vitro esophageal stratified epithelial layer model, and reported that they induced delocalization of CLDN4 and a decrease in the detergent-insoluble fraction of CLDN4 [15]. A recent paper showed that mice deficient in $\mathrm{Nrf} 2$, a transcription factor related to oxidative stress, showed a loss of esophageal barrier function and a decrease in CLDN4 expression [30]. In that report, even though nuclear localization of Nrf2 in the granular layer in GERD was shown, the level of CLDN4 assayed was focused on the total amount in the mucosa, and CLDN4 staining was focused on the basal layers. Therefore, further studies should be performed to determine the interaction of Nrf2 and CLDN4 in humans.

In an analysis of esophageal biopsy specimens using an Ussing chamber, the resistance of esophageal mucosa was lower in patients with GERD than in healthy controls, indicating a loss of barrier function in GERD [31-33]. Although no change in occludin or ZO-1 expression has been reported in GERD patients, the expression of CLDN1 and CLDN2 mRNA and protein has been reported to be increased [34] and unchanged [35] in GERD, and the expression of CLDN1 and CLDN4 has been reported to be decreased in GERD [33]. The reason for these inconsistent results may be due to the fact that total CLDN protein was measured rather than a specific subpopulation of CLDNs. As stated above, the total expression of TJ proteins themselves is not directly correlated to barrier function and TJ strand formation. Therefore, CLDNs contributing to TJ strand formation should be carefully analyzed in future studies. We recently found that the dot staining of CLDN4 in granular layers of esophageal epithelium is delocalized in GERD.

A recent report indicated that basal impedance measurement was useful for the analysis of esophageal barrier function. This basal impedance can be analyzed from the data of 24-h ambulatory esophageal impedance-pH monitoring, and the value of basal impedance is decreased in GERD [36, 37]. A more recent work showed that a probe inserted through endoscopy could measure mucosal resistance in a short time and that research groups are trying to establish an easy method for measuring mucosal barrier function [38]. A device for measuring mucosal conductance using a probe inserted through endoscopy is also being developed in Japan, a [39]. Such easy procedures for measuring mucosal barrier function are expected to provide a good tool for the diagnosis of NERD and the effect of GERD treatment. 


\section{Barrett's esophagus and esophageal cancer}

TJ proteins of esophageal squamous cell carcinoma and Barrett's esophagus and adenocarcinoma have also been analyzed. When the expression of TJ proteins in esophageal cancer are analyzed, the background expression pattern of TJ proteins should be precisely considered, because the expression patterns are totally different in stratified and columnar cells. Therefore, different TJ expression patterns should be compared to each appropriate background mucosa. Furthermore, the barrier function under normal conditions is different in the esophagus, stomach, and intestine, and these background differences should also be carefully taken into account when analyzing cancer and TJ proteins.

Few studies have analyzed TJ protein expression in esophageal squamous cell carcinoma; an increase in CLDN1 immunohistochemical staining, and no change in CLDN4 and CLDN7 staining, have been reported [40]. Immunohistochemical analysis indicated that CLDN2 expression was increased in Barrett's esophageal cancer and that CLDN3 and CLDN4 were up-regulated in Barrett's esophagus and adenocarcinoma [40]. In contrast, another report showed upregulation of CLDN2 and CLDN3 and downregulation of CLDN1 in Barrett's esophagus [41]. CLDN18, which has been reported to be specifically expressed in the stomach and lung, is also expressed in Barrett's esophagus. These data are consistent with the findings that Barrett's esophagus expresses not only intestinal phenotype markers but also gastric phenotype markers [42]. As the previous studies did not include precise analyses, further research is needed to determine the function of TJ proteins in Barrett's esophagus and cancer.

\section{Eosinophilic esophagitis (EoE)}

EoE is an allergy-mediated disease in which food antigens in contact with esophageal mucosa generate a T-helper cell type 2 (Th2) cytokine activation, and EoE is characterized by esophageal intraepithelial eosinophil infiltration [43]. It was recently reported that esophageal mucosal barrier function is disrupted even in EoE, and that esophageal mucosal impedance and DIS are related to the activity of EoE [44]. However, whether this barrier disruption is one of the causes of EoE or is a consequence of allergy is not clear.

Since the esophageal mucosal barrier is disrupted in EoE, the expression of TJ proteins in the esophagus was evaluated, and CLDN1 expression that was evaluated using immunohistochemistry was down-regulated [45, 46]. The barrier function of esophageal mucosa in EoE might be regulated by $\mathrm{Th} 2$ cytokines and filaggrin [47, 48].
However, it is still not clear how TJs are modulated in and how they affect the pathophysiology of EoE.

\section{Gastric cancer}

Abnormal expression patterns of claudins have recently been reported in cancers of the breast, stomach, colon, kidney, liver, and biliary tract, and are related to the progression and prognosis of these cancers [49]. Although CLDNs were reported to be decreased in cancers, the phenotypes of the cells in some organs, including the stomach and colon, were changed during cancerization. Several CLDNs including CLDN3, 4, and 7 have been reported to be increased in gastric cancers [50]. Loss of compartmentalization allows paracellular leakage of oncogenic factors and nutrients. CLDNs expressed in cancers may have roles that are unrelated to $\mathrm{TJ}$ strand formation, because some of these CLDNs are often found on broad lateral membranes and in the cytoplasm of epithelial cells [51].

To evaluate the development and progression of gastric cancers, Helicobacter pylori infection should first be taken into account. Atrophic and intestinal metaplasia induced by Helicobacter pylori infection changes the TJ protein expression pattern from that of gastric-type claudins to that of intestinal-type claudins [50, 52-55]. Therefore, the interpretation of an increase or decrease in TJ proteins in cancer should be carefully analyzed, and may be primarily associated with the phenotype change of gastric epithelial cells rather than with cancer development or progression. Furthermore, gastric cancers are heterogeneous and include two major phenotypes, intestinal and diffuse types. These different cancer phenotypes should be analyzed separately because the types of claudins expressed are totally different [56-58].

CLDN1, 3, 4, and 7 were found to be up-regulated in intestinal metaplasia that could be a precancerous lesion [50]. This upregulation correlated with expression of the transcription factor $\mathrm{Cdx} 2$ and with intestinal mucin expression which are related to intestinal metaplasia [50, 54, 59]. These data also indicate that expression of these CLDNs is not the primary cause of gastric cancer development [60].

CLDN1, 3, 4, 6, and 7 were up-regulated in intestinaltype gastric cancers [51, 61, 62], and CLDN18 was downregulated [53]. It should be noted that the up-regulated CLDN proteins are not located at TJs but are expressed in the cytoplasm and on lateral membranes, indicating that the up-regulated CLDNs do not function in TJ formation.

At this time, it is difficult to interpret all of the data related to CLDNs and TJ molecules of gastric cancers in terms of a unified hypothesis. However, there is one direction for the up-regulation in the progress [63] and for 
the downregulation in the malignant potential that can be explained by the loss of cell-cell association during invasion $[50,56]$. We recently reported that the downregulation of CLDN3 was associated with the proliferative potential of early gastric cancers [50]. CLDN1 was down-regulated in the metastasis of the cancer compared to the gastric cancer origin [64]. These data indicate promotion of cellcell dissociation by a decrease in CLDN expression. This possibility is supported by a report that the decrease in junctional CLDN3 and 7 at the submucosal invasive front of gastric cancer was significantly lower than that in the mucosal regions [50]. Another report indicated that neither CLDN3, 4, nor 18 was expressed at the invasive front [54]. Furthermore, CLDN3 and 4 are reportedly not expressed in cancer cells that invade the stroma individually or in clusters of cancer cells [65].

Additional studies are needed to elucidate whether these changes are potential biomarkers of cancer progression and prognosis. TJ molecules are also attractive potential targets of cancer treatment.

\section{Colon cancer}

Although the role of adherens junction proteins in colorectal cancer (CRC) has been studied extensively [66], the roles of TJ proteins are less well understood. The combination of expressed TJ proteins is different in different organs. Normal colonic mucosa is positive for CLDN1, 3, 4, 7, 8, 15, and 23 (Fig. 1). CLDN1, which is one of the genes regulated by beta-catenin/T-cell factor (Tcf)/lymphoid enhancer factor (LEF), was reported to be up-regulated in colon cancer [67]. CLDN1 expression was upregulated at all cell-cell boundaries and in the cytoplasm of cancer cells. This localization is in contrast to the normal dense localization of CLDN1 at apical borders of lateral membranes, and indicate that CLDN1 expression in itself does not mean that the expressed CLDN1 is functional at $\mathrm{TJ}$, and thus the broad lateral membrane expression of CLDN1 might have other roles in colorectal tumorigenesis. CLDN1 has also been reported to be a strong predictor of disease recurrence and poor survival [68]. Studies have noted that CLDN1 affects matrix metalloproteinase (MMP) activation [69] and frequent nuclear localization of CLDN1 in colon cancer, and that CLDN1 mediated changes in cellular phenotype with structural and functional changes in markers of epithelial-mesenchymal transition [70].

Upregulation of CLDN2 and down-regulation of CLDN4 and 7 have also been reported in colon cancers [71]. Some have proposed that these proteins are regulated by epidermal growth factor receptor activation signaling and promoter hypermethylation [72, 73].

Although CLDN18 is specifically expressed in normal gastric mucosa in the GI tract, it also expressed in some resected colon cancers, and its expression is related to expression of the gastric phenotype marker MUC5AC and to downregulation of CDX2. In CLDN18-expressing cases, the pathology of colon cancer is similar to that of gastric cancers, and CLDN18 expression is related to poor survival [74]. CLDN18 expression has also been reported to be associated with serrated adenocarcinoma of the colon [75]. These data indicate that aberrant expression of $\mathrm{TJ}$ proteins and of phenotype markers is strongly related to the progression and poor prognosis of cancers. TJ proteins are possible biomarkers of prognosis and of differential diagnosis, and are attractive targets for estimating the prognosis of patients.

\section{Irritable bowel syndrome (IBS)}

Functional gastrointestinal disorders are represented by functional dyspepsia (FD) and IBS. Disruption of duodenal and intestinal permeability has been reported in some cases of FD and IBS [76-80]. An interesting finding was that a fecal supernatant from either diarrhea-predominant or constipation-predominant IBS increased intestinal permeability [81-84], which shows that soluble mediators are involved in the increased permeability. Whether the increased permeability or the result of low-grade inflammation in the mucosa is the cause is still under debate. Nevertheless, regulation of the barrier dysfunction could maintain the barrier and change the immune-mediated inflammation cascade.

Reported changes in junctional proteins in IBS are summarized in Table 1. One study reported that the occludin protein was decreased in the colonic mucosa of IBS patients [82]. Another report, however, indicated that occludin and CLDN1 proteins were reduced in IBS with predominant diarrhea (IBS-D) but not in IBS with predominant constipation (IBS-C) or with constipation alternating with diarrhea (IBS-A), although the occludin and CLDN1 mRNA levels were similar among IBS subtypes and controls [83]. The mRNA levels of TJ-related molecules are still controversial, and further evaluations are warranted [84].

Intestinal permeability is increased in post-infectious IBS (PI-IBS) patients [85, 86]. The changes associated with such increased permeability must be preceded by a lack of recovery from the disruption of the TJ that occurred during acute infection. Genetic variants of molecules including TLR9, CDH1, and IL-6 have been reported to be independent risk factors for PI-IBS [87].

Stress induces gastrointestinal permeability through corticotrophin-releasing hormone (CRH). CRH increases permeability by stimulating the release of mediators, including tryptase from mast cells. The severity of psychological stress and the number of mucosal mast cells 
Table 1 Changes in the expression of junctional proteins in IBS

\begin{tabular}{|c|c|c|c|c|c|}
\hline & & IBS-total & IBS-D & IBS-A & IBS-C \\
\hline \multirow[t]{2}{*}{ Occludin } & Protein & Down (w) [82] & $\begin{array}{l}\text { Down (w) [83] } \\
\text { NC (w) [79] } \\
\text { Down (p-occl w) [79] } \\
\text { Down (h) [79] }\end{array}$ & $\mathrm{NC}(w)$ [83] & $\mathrm{NC}(\mathrm{w})$ [83] \\
\hline & mRNA & $\begin{array}{l}\mathrm{NC}[82] \\
\mathrm{NC}[81]\end{array}$ & Down [84] & Down [84] & Down [84] \\
\hline \multirow[t]{2}{*}{ ZO-1 } & Protein & Down (w) [83] & $\begin{array}{l}\mathrm{NC}(\mathrm{w})[83] \\
\text { Down (h) [93] }\end{array}$ & $\begin{array}{l}\mathrm{NC}(\mathrm{w})[83] \\
\text { Down (h) [92] }\end{array}$ & $\mathrm{NC}(\mathrm{w})$ [83] \\
\hline & mRNA & Down [81] & $\begin{array}{l}\text { Down [93] } \\
\text { Down [84] }\end{array}$ & Down [84] & Down [84] \\
\hline $\mathrm{ZO}-2$ & $\begin{array}{l}\text { Protein } \\
\text { mRNA }\end{array}$ & & $\begin{array}{l}\text { Down (h) [93] } \\
\text { NC [93] }\end{array}$ & & \\
\hline $\mathrm{ZO}-3$ & $\begin{array}{l}\text { Protein } \\
\text { mRNA }\end{array}$ & & $\begin{array}{l}\text { NC (h) [93] } \\
\text { Down [93] }\end{array}$ & & \\
\hline JAM-A & $\begin{array}{l}\text { Protein } \\
\text { mRNA }\end{array}$ & & Down (h) [91] & Down (h) [91] & \\
\hline CLDN1 & Protein & $\mathrm{NC}(\mathrm{w})$ [83] & $\mathrm{NC}(\mathrm{w})$ [79] & $\begin{array}{l}\mathrm{NC}(\mathrm{w})[83] \\
\mathrm{NC}(\mathrm{h})[92]\end{array}$ & $\mathrm{NC}(\mathrm{w})$ [83] \\
\hline & mRNA & & & & \\
\hline CLDN2 & $\begin{array}{l}\text { Protein } \\
\text { mRNA }\end{array}$ & & Up (w) [79] & & \\
\hline CLDN3 & $\begin{array}{l}\text { Protein } \\
\text { mRNA }\end{array}$ & & $\mathrm{NC}(\mathrm{w})$ [79] & & \\
\hline CLDN4 & $\begin{array}{l}\text { Protein } \\
\text { mRNA }\end{array}$ & & $\mathrm{NC}(w)$ [79] & & \\
\hline E-cadherin & $\begin{array}{l}\text { Protein } \\
\text { mRNA }\end{array}$ & & $\mathrm{NC}(\mathrm{h})$ [92] & Down (h) [92] & \\
\hline
\end{tabular}

$I B S$ irritable bowel syndrome, IBS-D IBS with predominant diarrhea, IBS- $A$ IBS with constipation alternating with diarrhea, IBS-C IBS with predominant constipation, $N C$ no change, $w$ western blot, $h$ immunohistochemistry, $p$-occl phospho-occludin

Site of biopsies: [79, 93], jejunum; [81], not mentioned; [82, 83], descending colon; [84, 91, 92], cecum were found to be correlated in IBS patients regardless of the subtypes [88]. The increased permeability was blocked by the mast cell stabilizer lodoxamide or by disodium cromoglycate pretreatment $[89,90]$, indicating the importance of mediators released from infiltrated mast cells in intestinal permeability.

In IBS-D and IBS-A patients, colonic JAM-A expression was found to be decreased and the degree of abdominal pain correlated with the decrease in JAM-A. Furthermore, tryptase increased intestinal permeability and decreased expression of JAM-A, CLDN1, and ZO-1 in vitro [91]. The same group also reported that $\mathrm{ZO}-1$ and E-cadherin expression were significantly lower in the surface epithelium of the IBS group (IBS-D and IBS-A) [92]. The expression of ZO-1 was decreased in IBS-D at both the gene and the protein level, with protein redistribution from the TJ to the cytoplasm [93].
The adherens junction protein E-cadherin was also reportedly decreased in IBS-D and IBS-A, and a singlenucleotide polymorphism of the CDH1 gene encoding E-cadherin was associated with PI-IBS [87]. IBS-D patients also exhibited increased protein expression of CLDN2 in the jejunum [79].

MiR-29a was increased in IBS-D patients, with increased intestinal permeability. The effect of MiR-29a on intestinal permeability might be due to the regulation of glutamine synthetase [94]. MiR-29a and MiR-29b also reduced the levels of CLDN1 mRNA and increased intestinal permeability [95]. These data indicate that strategies to block MiR29 might be a good way to restore intestinal permeability in IBS-D. Recent data related to the treatment of IBS also indicated that glutamine increased CLDN1 expression in the colonic mucosa in IBS-D [96]. Glutamine supplementation may be beneficial in IBS-D 
patients to reduce intestinal hyperpermeability and visceral hypersensitivity. However, a better glutamine delivery method is still needed, since glutamine is rapidly absorbed in the small intestine [97].

\section{Inflammatory bowel disease (IBD)}

IBD is a chronic intestinal inflammatory condition with unknown etiology. The intestinal mucosal barrier is important in the regulation of the chaotic invasion of bacterial flora food antigens from the mucosal surface [98, 99]. The regulation of humoral factors from the luminal side is also important in controlling the immune system in the intestine. Recent genome-wide association studies have revealed ulcerative colitis (UC)-susceptible single-nucleotide polymorphisms in genes that might be involved in intestinal barrier function, including $H N F 4, C D H 1$, and $L A M B 1$ [100]. E-cadherin, which is encoded by $C D H 1$, is an adherens junction protein that regulates strong cell-cell adhesion, and E-cadherin deficiency was found to aggravate colitis in a mouse model [101]. However, these data are still crude, and functional analysis of these molecules in terms of the development of UC is still immature and inconclusive [102]. Analysis of claudins in the intestine demonstrated that intestine-specific CLDN7 deficiency resulted in intestinal inflammation 3 weeks after birth and increased paracellular permeability 2 weeks after birth [103]. Intestine-specific CLDN7 deficiency also enhanced the paracellular permeability for fMLP, a major bacterial product, following which colonic inflammation was initiated [103]. JAM-A-deficient mice also exhibited susceptibility to dextran sulfate sodium and developed a more severe colitis than control mice [104, 105]. Such data indicate that $\mathrm{TJ}$ proteins are important for intestinal barrier function and that their loss increases intestinal permeability. At the same time, however, disruption of barrier function alone is not always sufficient to cause IBD.

In observational studies in IBD patients, CLDN4 and CLDN7 were down-regulated and CLDN2 was up-regulated in UC patients [106]. JAM-A expression was downregulated in UC and in Crohn's disease (CD) at the inflamed mucosa [105]. In contrast, CLDN1 and CLDN2 were reported to be increased in UC and CD [107]. Occludin and CLDN8 were down-regulated and CLDN2 was up-regulated in patients with mild to moderate $\mathrm{CD}$ [108]. These changes might be mediated by inflammatory cytokines, because TNF $\alpha$ and IFN $\gamma$ change the barrier function through MLCK activation [109], and TNF $\alpha$ increases CLDN2 expression [110]. In CD patients, anti$\mathrm{TNF} \alpha$ antibody not only regulates inflammation but also improves intestinal mucosal permeability [111]. Low-dose sulfasalazine was also shown to prevent TNF $\alpha$-induced TJ disruption [109]. As stated above, the CLDN changes are not limited to the apical lateral membrane of colonic epithelial cells where TJs exist. CLDNs expressed on broad lateral membranes are affected, and this may lead to neoplastic changes in inflammatory mucosal cells.

At this time, it is clear that preventing inflammation introduces an improvement in intestinal barrier function. Although it remains unclear whether the epithelial barrier dysfunction is a cause or a consequence of intestinal inflammation in IBD, these findings suggest that intestinal epithelial TJs can still be targets for enhancing the integrity of the intestinal barrier in IBD [112]. Glutamine is an important luminal component that preserves intestinal barrier function, and glutamine administration for $\mathrm{CD}$ patients has been reported in two small studies. Although the data were negative, adequately powered randomized controlled studies are needed before definitive conclusions can be reached regarding the effect of glutamine in patients with CD [113].

\section{Conclusion}

Recent studies of TJ have revealed the localization and the modulation of these TJs and related proteins in gastrointestinal diseases, and these changes represent potential biomarkers of each disease and targets for treatment. However, treatments directly targeting the regulation of TJ and TJ proteins have yet to be developed. Further studies are needed to devise new treatment strategies by targeting TJs in gastrointestinal diseases.

\section{Compliance with ethical standards}

Conflict of interest The authors declare that they have no conflict of interest.

\section{References}

1. Tsukita S, Furuse M. Pores in the wall: claudins constitute tight junction strands containing aqueous pores. J Cell Biol. 2000;149:13-6.

2. Tsukita S, Furuse M. The structure and function of claudins, cell adhesion molecules at tight junctions. Ann N Y Acad Sci. 2000;915:129-35.

3. Stevenson BR, Siliciano JD, Mooseker MS, et al. Identification of ZO-1: a high molecular weight polypeptide associated with the tight junction (zonula occludens) in a variety of epithelia. J Cell Biol. 1986;103:755-66.

4. Tsukita S, Furuse M, Itoh M. Multifunctional strands in tight junctions. Nat Rev Mol Cell Biol. 2001;2:285-93.

5. Saitou M, Furuse M, Sasaki H, et al. Complex phenotype of mice lacking occludin, a component of tight junction strands. Mol Biol Cell. 2000;11:4131-42.

6. Furuse M, Hata M, Furuse K, et al. Claudin-based tight junctions are crucial for the mammalian epidermal barrier: a lesson from claudin-1-deficient mice. J Cell Biol. 2002;156:1099-111. 
7. Schulzke JD, Gitter AH, Mankertz J, et al. Epithelial transport and barrier function in occludin-deficient mice. Biochim Biophys Acta. 2005;1669:34-42.

8. Mineta K, Yamamoto Y, Yamazaki Y, et al. Predicted expansion of the claudin multigene family. FEBS Lett. 2011;585:606-12.

9. Krause G, Winkler L, Mueller SL, et al. Structure and function of claudins. Biochim Biophys Acta. 2008;1778:631-45.

10. Swisshelm K, Macek R, Kubbies M. Role of claudins in tumorigenesis. Adv Drug Deliv Rev. 2005;57:919-28.

11. Fawcett DW. Epithelium. In: Fawcett DW, William B, editors. A textbook of histology. 12th ed. New York: Chapman \& Hall; 1994. p. 57-83.

12. Chen X, Oshima T, Tomita T, et al. Acidic bile salts modulate the squamous epithelial barrier function by modulating tight junction proteins. Am J Physiol Gastrointest Liver Physiol. 2011;301:G203-9.

13. Oshima T, Gedda K, Koseki J, et al. Establishment of esophageal-like non-keratinized stratified epithelium using normal human bronchial epithelial cells. Am J Physiol Cell Physiol. 2011;300:C1422-9.

14. Oshima T, Koseki J, Chen X, et al. Acid modulates the squamous epithelial barrier function by modulating the localization of claudins in the superficial layers. Lab Invest. 2012;92:22-31.

15. Chen X, Oshima T, Shan J, et al. Bile salts disrupt human esophageal squamous epithelial barrier function by modulating tight junction proteins. Am J Physiol Gastrointest Liver Physiol. 2012;303:G199-208.

16. Shan J, Oshima T, Muto T, et al. Epithelial-derived nuclear IL33 aggravates inflammation in the pathogenesis of reflux esophagitis. J Gastroenterol. 2015;50:414-23.

17. Wu L, Oshima T, Tomita T, et al. Serotonin disrupts esophageal mucosal integrity: an investigation using a stratified squamous epithelial model. J Gastroenterol. 2016. doi:10.1007/s00535016-1195-z.

18. Baker M. Reproducibility crisis: blame it on the antibodies. Nature. 2015;521:274-6.

19. Asaoka D, Miwa H, Hirai S, et al. Altered localization and expression of tight-junction proteins in a rat model with chronic acid reflux esophagitis. J Gastroenterol. 2005;40:781-90.

20. Miwa H, Oshima T, Sakurai J, et al. Experimental oesophagitis in the rat is associated with decreased voluntary movement. Neurogastroenterol Motil. 2009;21:296-303.

21. Miwa H, Koseki J, Oshima T, et al. Rikkunshito, a traditional Japanese medicine, may relieve abdominal symptoms in rats with experimental esophagitis by improving the barrier function of epithelial cells in esophageal mucosa. J Gastroenterol. 2010;45:478-87.

22. Oguro M, Koike M, Ueno T, et al. Dissociation and dispersion of claudin-3 from the tight junction could be one of the most sensitive indicators of reflux esophagitis in a rat model of the disease. J Gastroenterol. 2011;46:629-38.

23. Oshima T, Sasaki M, Kataoka H, et al. Wip1 protects hydrogen peroxide-induced colonic epithelial barrier dysfunction. Cell Mol Life Sci. 2007;64:3139-47.

24. van Malenstein H, Farre R, Sifrim D. Esophageal dilated intercellular spaces (DIS) and nonerosive reflux disease. Am J Gastroenterol. 2008;103:1021-8.

25. Caviglia R, Ribolsi M, Maggiano N, et al. Dilated intercellular spaces of esophageal epithelium in nonerosive reflux disease patients with physiological esophageal acid exposure. Am J Gastroenterol. 2005;100:543-8.

26. Vela MF, Craft BM, Sharma N, et al. Refractory heartburn: comparison of intercellular space diameter in documented GERD vs. functional heartburn. Am J Gastroenterol. 2011;106:844-50.
27. Kandulski A, Jechorek D, Caro C, et al. Histomorphological differentiation of non-erosive reflux disease and functional heartburn in patients with PPI-refractory heartburn. Aliment Pharmacol Ther. 2013;38:643-51.

28. Farre R, Fornari F, Blondeau K, et al. Acid and weakly acidic solutions impair mucosal integrity of distal exposed and proximal non-exposed human oesophagus. Gut. 2010;59:164-9.

29. Farre R, De Vos R, Geboes K, et al. Critical role of stress in increased oesophageal mucosa permeability and dilated intercellular spaces. Gut. 2007;56:1191-7.

30. Chen H, Hu Y, Fang Y, et al. Nrf2 deficiency impairs the barrier function of mouse oesophageal epithelium. Gut. 2014;63:711-9.

31. Farre R, van Malenstein H, De Vos R, et al. Short exposure of oesophageal mucosa to bile acids, both in acidic and weakly acidic conditions, can impair mucosal integrity and provoke dilated intercellular spaces. Gut. 2008;57:1366-74.

32. Jovov B, Que J, Tobey NA, et al. Role of E-cadherin in the pathogenesis of gastroesophageal reflux disease. Am J Gastroenterol. 2011;106:1039-47.

33. Bjorkman EV, Edebo A, Oltean M, et al. Esophageal barrier function and tight junction expression in healthy subjects and patients with gastroesophageal reflux disease: functionality of esophageal mucosa exposed to bile salt and trypsin in vitro. Scand J Gastroenterol. 2013;48:1118-26.

34. Monkemuller K, Wex T, Kuester D, et al. Role of tight junction proteins in gastroesophageal reflux disease. BMC Gastroenterol. 2012;12:128.

35. Liu CC, Lee JW, Liu TT, et al. Relevance of ultrastructural alterations of intercellular junction morphology in inflamed human esophagus. J Neurogastroenterol Motil. 2013;19:324-31.

36. Farre R, Blondeau K, Clement D, et al. Evaluation of oesophageal mucosa integrity by the intraluminal impedance technique. Gut. 2011;60:885-92.

37. Kessing BF, Bredenoord AJ, Weijenborg PW, et al. Esophageal acid exposure decreases intraluminal baseline impedance levels. Am J Gastroenterol. 2011;106:2093-7.

38. Ates F, Yuksel ES, Higginbotham T, et al. Mucosal impedance discriminates GERD from non-GERD conditions. Gastroenterology. 2015;148:334-43.

39. Koizumi H, Suzuki H, Ohbuchi T, et al. Increased permeability of the epithelium of middle ear cholesteatoma. Clin Otolaryngol. 2015;40:106-14.

40. Gyorffy H, Holczbauer A, Nagy P, et al. Claudin expression in Barrett's esophagus and adenocarcinoma. Virchows Arch. 2005;447:961-8.

41. Mullin JM, Valenzano MC, Trembeth S, et al. Transepithelial leak in Barrett's esophagus. Dig Dis Sci. 2006;51:2326-36.

42. Arul GS, Moorghen M, Myerscough N, et al. Mucin gene expression in Barrett's oesophagus: an in situ hybridisation and immunohistochemical study. Gut. 2000;47:753-61.

43. Liacouras CA, Furuta GT, Hirano I, et al. Eosinophilic esophagitis: updated consensus recommendations for children and adults. J Allergy Clin Immunol. 2011;128:3-20 e6; quiz 21-2.

44. Katzka DA, Ravi K, Geno DM, et al. Endoscopic mucosal impedance measurements correlate with eosinophilia and dilation of intercellular spaces in patients with eosinophilic esophagitis. Clin Gastroenterol Hepatol. 2015;13:1242-8 e1.

45. Abdulnour-Nakhoul SM, Al-Tawil Y, Gyftopoulos AA, et al. Alterations in junctional proteins, inflammatory mediators and extracellular matrix molecules in eosinophilic esophagitis. Clin Immunol. 2013;148:265-78.

46. Katzka DA, Tadi R, Smyrk TC, et al. Effects of topical steroids on tight junction proteins and spongiosis in esophageal epithelia of patients with eosinophilic esophagitis. Clin Gastroenterol Hepatol. 2014;12:1824-9 e1. 
47. Blanchard C, Stucke EM, Burwinkel K, et al. Coordinate interaction between IL-13 and epithelial differentiation cluster genes in eosinophilic esophagitis. J Immunol. 2010;184:4033-41.

48. van Rhijn BD, Verheij J, van den Bergh Weerman MA, et al. Histological response to fluticasone propionate in patients with eosinophilic esophagitis is associated with improved functional esophageal mucosal integrity. Am J Gastroenterol. 2015;110:1289-97.

49. Ouban A, Ahmed AA. Claudins in human cancer: a review. Histol Histopathol. 2010;25:83-90.

50. Okugawa T, Oshima T, Chen X, et al. Down-regulation of claudin-3 is associated with proliferative potential in early gastric cancers. Dig Dis Sci. 2012;57:1562-7.

51. Oliveira SS, Morgado-Diaz JA. Claudins: multifunctional players in epithelial tight junctions and their role in cancer. Cell Mol Life Sci. 2007;64:17-28.

52. Hayashi D, Tamura A, Tanaka H, et al. Deficiency of claudin-18 causes paracellular $\mathrm{H}+$ leakage, up-regulation of interleukin1beta, and atrophic gastritis in mice. Gastroenterology. 2012;142:292-304.

53. Oshima T, Shan J, Okugawa T, et al. Down-regulation of claudin-18 is associated with the proliferative and invasive potential of gastric cancer at the invasive front. PLoS One. 2013;8:e74757.

54. Matsuda Y, Semba S, Ueda J, et al. Gastric and intestinal claudin expression at the invasive front of gastric carcinoma. Cancer Sci. 2007;98:1014-9.

55. Sanada Y, Oue N, Mitani Y, et al. Down-regulation of the claudin-18 gene, identified through serial analysis of gene expression data analysis, in gastric cancer with an intestinal phenotype. J Pathol. 2006;208:633-42.

56. Kuo WL, Lee LY, Wu CM, et al. Differential expression of claudin- 4 between intestinal and diffuse-type gastric cancer. Oncol Rep. 2006;16:729-34.

57. Wu CM, Lee YS, Wang TH, et al. Identification of differential gene expression between intestinal and diffuse gastric cancer using cDNA microarray. Oncol Rep. 2006;15:57-64.

58. Park JY, Park KH, Oh TY, et al. Up-regulated claudin 7 expression in intestinal-type gastric carcinoma. Oncol Rep. 2007; 18:377-82.

59. Soini Y, Tommola S, Helin H, et al. Claudins 1, 3, 4 and 5 in gastric carcinoma, loss of claudin expression associates with the diffuse subtype. Virchows Arch. 2006;448:52-8.

60. Ohtani S, Terashima M, Satoh J, et al. Expression of tightjunction-associated proteins in human gastric cancer: downregulation of claudin- 4 correlates with tumor aggressiveness and survival. Gastric Cancer. 2009;12:43-51.

61. Resnick MB, Gavilanez M, Newton E, et al. Claudin expression in gastric adenocarcinomas: a tissue microarray study with prognostic correlation. Hum Pathol. 2005;36:886-92.

62. Johnson AH, Frierson HF, Zaika A, et al. Expression of tightjunction protein claudin-7 is an early event in gastric tumorigenesis. Am J Pathol. 2005;167:577-84.

63. Ikeo K, Oshima T, Shan J, et al. Junctional adhesion molecule-A promotes proliferation and inhibits apoptosis of gastric cancer. Hepatogastroenterology. 2015;62:540-5.

64. Jung H, Jun KH, Jung JH, et al. The expression of claudin-1, claudin-2, claudin-3, and claudin-4 in gastric cancer tissue. J Surg Res. 2011;167:e185-91.

65. Kamata I, Ishikawa Y, Akishima-Fukasawa Y, et al. Significance of lymphatic invasion and cancer invasion-related proteins on lymph node metastasis in gastric cancer. J Gastroenterol Hepatol. 2009;24:1527-33.

66. Kim SA, Inamura K, Yamauchi M, et al. Loss of CDH1 (Ecadherin) expression is associated with infiltrative tumour growth and lymph node metastasis. $\mathrm{Br} \mathrm{J}$ Cancer. 2016;114:199-206.

67. Miwa N, Furuse M, Tsukita S, et al. Involvement of claudin-1 in the beta-catenin/Tcf signaling pathway and its frequent upregulation in human colorectal cancers. Oncol Res. 2001;12:469-76.

68. Resnick MB, Konkin T, Routhier J, et al. Claudin-1 is a strong prognostic indicator in stage II colonic cancer: a tissue microarray study. Mod Pathol. 2005;18:511-8.

69. Miyamori H, Takino T, Kobayashi Y, et al. Claudin promotes activation of pro-matrix metalloproteinase- 2 mediated by membrane-type matrix metalloproteinases. J Biol Chem. 2001;276:28204-11.

70. Dhawan P, Singh AB, Deane NG, et al. Claudin-1 regulates cellular transformation and metastatic behavior in colon cancer. J Clin Invest. 2005;115:1765-76.

71. Ueda J, Semba S, Chiba H, et al. Heterogeneous expression of claudin-4 in human colorectal cancer: decreased claudin-4 expression at the invasive front correlates cancer invasion and metastasis. Pathobiology. 2007;74:32-41.

72. Dhawan P, Ahmad R, Chaturvedi R, et al. Claudin-2 expression increases tumorigenicity of colon cancer cells: role of epidermal growth factor receptor activation. Oncogene. 2011;30:3234-47.

73. Nakayama F, Semba S, Usami Y, et al. Hypermethylationmodulated downregulation of claudin-7 expression promotes the progression of colorectal carcinoma. Pathobiology. 2008;75:177-85.

74. Matsuda M, Sentani K, Noguchi T, et al. Immunohistochemical analysis of colorectal cancer with gastric phenotype: claudin-18 is associated with poor prognosis. Pathol Int. 2010;60:673-80.

75. Sentani K, Sakamoto N, Shimamoto F, et al. Expression of olfactomedin 4 and claudin-18 in serrated neoplasia of the colorectum: a characteristic pattern is associated with sessile serrated lesion. Histopathology. 2013;62:1018-27.

76. Vanheel H, Vicario M, Vanuytsel T, et al. Impaired duodenal mucosal integrity and low-grade inflammation in functional dyspepsia. Gut. 2014;63:262-71.

77. Dunlop SP, Hebden J, Campbell E, et al. Abnormal intestinal permeability in subgroups of diarrhea-predominant irritable bowel syndromes. Am J Gastroenterol. 2006;101:1288-94.

78. Gecse K, Roka R, Sera T, et al. Leaky gut in patients with diarrhea-predominant irritable bowel syndrome and inactive ulcerative colitis. Digestion. 2012;85:40-6.

79. Martinez C, Lobo B, Pigrau M, et al. Diarrhoea-predominant irritable bowel syndrome: an organic disorder with structural abnormalities in the jejunal epithelial barrier. Gut. 2013;62:1160-8.

80. Fukudo S, Kaneko H, Akiho H, et al. Evidence-based clinical practice guidelines for irritable bowel syndrome. J Gastroenterol. 2015;50:11-30.

81. Piche T, Barbara G, Aubert P, et al. Impaired intestinal barrier integrity in the colon of patients with irritable bowel syndrome: involvement of soluble mediators. Gut. 2009;58:196-201.

82. Coeffier M, Gloro R, Boukhettala N, et al. Increased proteasome-mediated degradation of occludin in irritable bowel syndrome. Am J Gastroenterol. 2010;105:1181-8.

83. Bertiaux-Vandaele N, Youmba SB, Belmonte L, et al. The expression and the cellular distribution of the tight junction proteins are altered in irritable bowel syndrome patients with differences according to the disease subtype. Am J Gastroenterol. 2011;106:2165-73.

84. Vivinus-Nebot M, Frin-Mathy G, Bzioueche H, et al. Functional bowel symptoms in quiescent inflammatory bowel diseases: role of epithelial barrier disruption and low-grade inflammation. Gut. 2014;63:744-52.

85. Spiller RC, Jenkins D, Thornley JP, et al. Increased rectal mucosal enteroendocrine cells, $\mathrm{T}$ lymphocytes, and increased 
gut permeability following acute Campylobacter enteritis and in post-dysenteric irritable bowel syndrome. Gut. 2000;47:804-11.

86. Marshall JK, Thabane M, Garg AX, et al. Intestinal permeability in patients with irritable bowel syndrome after a waterborne outbreak of acute gastroenteritis in Walkerton, Ontario. Aliment Pharmacol Ther. 2004;20:1317-22.

87. Villani AC, Lemire M, Thabane M, et al. Genetic risk factors for post-infectious irritable bowel syndrome following a waterborne outbreak of gastroenteritis. Gastroenterology. 2010;138:1502-13.

88. Piche T, Saint-Paul MC, Dainese R, et al. Mast cells and cellularity of the colonic mucosa correlated with fatigue and depression in irritable bowel syndrome. Gut. 2008;57:468-73.

89. Vanuytsel T, van Wanrooy S, Vanheel H, et al. Psychological stress and corticotropin-releasing hormone increase intestinal permeability in humans by a mast cell-dependent mechanism. Gut. 2014;63:1293-9.

90. Wallon C, Yang PC, Keita AV, et al. Corticotropin-releasing hormone $(\mathrm{CRH})$ regulates macromolecular permeability via mast cells in normal human colonic biopsies in vitro. Gut. 2008;57:50-8.

91. Wilcz-Villega EM, McClean S, O'Sullivan MA. Mast cell tryptase reduces junctional adhesion molecule-A (JAM-A) expression in intestinal epithelial cells: implications for the mechanisms of barrier dysfunction in irritable bowel syndrome. Am J Gastroenterol. 2013;108:1140-51.

92. Wilcz-Villega E, McClean S, O'Sullivan M. Reduced E-cadherin expression is associated with abdominal pain and symptom duration in a study of alternating and diarrhea predominant IBS. Neurogastroenterol Motil. 2014;26:316-25.

93. Martinez C, Vicario M, Ramos L, et al. The jejunum of diarrheapredominant irritable bowel syndrome shows molecular alterations in the tight junction signaling pathway that are associated with mucosal pathobiology and clinical manifestations. Am J Gastroenterol. 2012;107:736-46.

94. Zhou Q, Souba WW, Croce CM, et al. MicroRNA-29a regulates intestinal membrane permeability in patients with irritable bowel syndrome. Gut. 2010;59:775-84.

95. Zhou Q, Costinean S, Croce CM, et al. MicroRNA 29 targets nuclear factor-kappaB-repressing factor and Claudin 1 to increase intestinal permeability. Gastroenterology. 2015;148:158-69 e8.

96. Bertrand J, Ghouzali I, Guérin C, et al. Glutamine restores tight junction protein claudin- 1 expression in colonic mucosa of patients with diarrhea-predominant irritable bowel syndrome. JPEN J Parenter Enteral Nutr. 2015. doi:10.1177/0148607115587330.

97. Coeffier M, Dechelotte P, Ducrotte P. Intestinal permeability in patients with diarrhea-predominant irritable bowel syndrome: is there a place for glutamine supplementation? Gastroenterology. 2015;148:1079-80.

98. Kawaguchi T, Mori M, Saito K, et al. Food antigen-induced immune responses in Crohn's disease patients and experimental colitis mice. J Gastroenterol. 2015;50:394-405.
99. Frehn L, Jansen A, Bennek E, et al. Distinct patterns of IgG and $\operatorname{IgA}$ against food and microbial antigens in serum and feces of patients with inflammatory bowel diseases. PLoS One. 2014;9:e106750.

100. Ventham NT, Kennedy NA, Nimmo ER, et al. Beyond gene discovery in inflammatory bowel disease: the emerging role of epigenetics. Gastroenterology. 2013;145:293-308.

101. Grill JI, Neumann J, Hiltwein F, et al. Intestinal E-cadherin deficiency aggravates dextran sodium sulfate-induced colitis. Dig Dis Sci. 2015;60:895-902.

102. Prager M, Buettner J, Buening C. Genes involved in the regulation of intestinal permeability and their role in ulcerative colitis. J Dig Dis. 2015;16:713-22.

103. Tanaka H, Takechi M, Kiyonari H, et al. Intestinal deletion of Claudin-7 enhances paracellular organic solute flux and initiates colonic inflammation in mice. Gut. 2015;64:1529-38.

104. Laukoetter MG, Nava P, Lee WY, et al. JAM-A regulates permeability and inflammation in the intestine in vivo. J Exp Med. 2007;204:3067-76.

105. Vetrano S, Rescigno M, Cera MR, et al. Unique role of junctional adhesion molecule-a in maintaining mucosal homeostasis in inflammatory bowel disease. Gastroenterology. 2008;135:173-84.

106. Oshima T, Miwa H, Joh T. Changes in the expression of claudins in active ulcerative colitis. J Gastroenterol Hepatol. 2008;23(Suppl 2):S146-50.

107. Weber CR, Nalle SC, Tretiakova M, et al. Claudin-1 and claudin-2 expression is elevated in inflammatory bowel disease and may contribute to early neoplastic transformation. Lab Invest. 2008;88:1110-20.

108. Zeissig S, Burgel N, Gunzel D, et al. Changes in expression and distribution of claudin 2, 5 and 8 lead to discontinuous tight junctions and barrier dysfunction in active Crohn's disease. Gut. 2007;56:61-72.

109. Wang F, Graham WV, Wang Y, et al. Interferon-gamma and tumor necrosis factor-alpha synergize to induce intestinal epithelial barrier dysfunction by up-regulating myosin light chain kinase expression. Am J Pathol. 2005;166:409-19.

110. Mankertz J, Schulzke JD. Altered permeability in inflammatory bowel disease: pathophysiology and clinical implications. Curr Opin Gastroenterol. 2007;23:379-83.

111. Suenaert P, Bulteel V, Lemmens L, et al. Anti-tumor necrosis factor treatment restores the gut barrier in Crohn's disease. Am J Gastroenterol. 2002;97:2000-4.

112. Bischoff SC, Barbara G, Buurman W, et al. Intestinal permeability: a new target for disease prevention and therapy. BMC Gastroenterol. 2014;14:189.

113. Akobeng AK, Elawad M, Gordon M. Glutamine for induction of remission in Crohn's disease. Cochrane Database Syst Rev. 2016;2:CD007348. 